\title{
Development of an expert system for abnormal operating procedures in a main control room
}

\author{
Min-Han Hsieh ${ }^{a}$, Sheue-Ling Hwang ${ }^{a}{ }^{*}$, Kang-Hong Liu ${ }^{a}$ Sheau-Farn Max Liang ${ }^{b}$, Chang-Fu Chuang ${ }^{c}$ \\ ${ }^{a}$ Institute of Industrial Engineering and Engineering Management, National Tsing Hua University, \\ Hsinchu 300, Taiwan \\ ${ }^{\mathrm{b}}$ Institute of Industrial Engineering and Management, National Taipei University of Technology, Taipei \\ 106 Taiwan \\ ${ }^{c}$ Atomic Energy Council, Taipei 234, Taiwan, ROC
}

\begin{abstract}
The study was conducted from the perspective of human factors engineering in order to compare the process that operators originally used to diagnose potential and actual faults with a process that included an expert system for diagnosing faults. The results of the study indicated that the existence of an expert system for fault diagnosis makes the task of fault diagnosis easier and reduces errors by quickly suggesting likely Abnormal Operating Procedures (AOPs)
\end{abstract}

Keywords: Nuclear Power Plant, Fault diagnosis, Expert system

\section{Introduction}

In order to maintain safety in NPPs, the human factor issue cannot be ignored. The Three Mile Island accident in 1979 in the USA and the Chernobyl disaster of 1986 in the Soviet Union were both caused by human errors that dealt with decision making, receiving information, and action selection. In a nuclear power plant, a large number of alarms often occur at the same time during an incident. In addition, the alarms come from many different systems. It make difficult for operator to diagnose efficiently. Too many information imposes a heavy burden on operators in a time-critical situation, and it is very difficult for them to conduct a thorough assessment of each individual symptom in a short period of time. Therefore, the process of decision-making during identifying AOPs is very complicated. How to help the operator diagnose and identify AOP accurately is an important issue.

Therefore, this study was undertaken to develop an expert system to reduce the complexity of the deci- sion-making process by aiding operators' cognitive activities, integrating unusual symptoms, and identifying the most suitable abnormal operating procedure (AOP) for operators.

\section{Backgrounds}

With the technical development of computer, human-machine systems are becoming increasingly automated. However, human operators are still important even with a high degree of automation in a nuclear power plant. The operators' tasks in a modern system include information gathering, planning, decision making, and avoiding unforeseen risks through the alarm system [1]. In the steady state, the operators' tasks are to monitor and maintain the system stable continually [2]. During abnormal situations, a well-trained operator should comprehend a malfunction in real time by analyzing alarms, assessing values, or recognizing unusual trends of multiple instruments. In addition, operators sometimes must foresee the possibility of unforeseen risks [3-4].

*Corresponding author. E-mail: slhwang@ie.nthu.edu.tw 
However, the automation of alarm system not only brings a lot of convenience, but also brings other problems. The operators must monitor a large number of information sources and make the system efficiently and safely in such complex automated systems. It is difficult for operators to identify abnormal situations with many alarms within a short time.

In the NPP, the operator must diagnose the cause when the plant is in the abnormal status. Isaac et al. [5] indicated that human error is a major contributor (70-90\%) to accidents at NPPs. Therefore, the correct decision-making is extremely important. Since the decision-making environment is extremely complicated and data intensive, the use of automated systems or expert systems to aid decision making is likely to become more common.

The main function of the expert system is to identify faults, to reduce human errors, to decrease the mental workload of operators in emergency and to avoid the occurrence of disaster. Artificial intelligence techniques have the potential to make a significant contribution to the reliable operation of NPPs, and there were many previous studies concerning the design and implementation of excellent expert systems. Kwon et al.[6] applied the Hidden Markov Model (HMM) to identify accidents in NPPs and showed its robustness. The accident identification system accurately identifies the type of accident and also predicts abnormal occurrences in advance. Lee et al.[7] developed the fault diagnosis advisory system (FDAS), which is based on dynamic neural networks. They indicated that FDAS facilitates the fault diagnosis task and reduces errors by quickly suggesting appropriate courses of action. FDAS provides accurate, reliable advice for operators who must make decisions quickly. The operators can identify the correct EOP (Emergency Operating Procedure) in real time. However, according to several papers that assessed the evaluation results provided by decision support systems, such systems do not guarantee improvement in the operator's performance. Some support systems could actually increase the operator's mental workload during these critical times [8-9]. Therefore, an effective expert system must aid, not hinder, the operator's cognitive processes.

Thus, an expert system should be capable of repeating and updating information continually and it should be capable of processing multiple problems simultaneously.

\section{Method}

After visiting the operation training in the Lungman nuclear power plant( a constructing NPP in Taiwan) for several times and the interviews with operators and experts, this study focuses on constructing a fault diagnosis support system to improve the performance of diagnosing unusual situation in NPPs and lower the chance of human errors. The experimental method was described in the following section. Figure 1 shows the flowchart for study process.

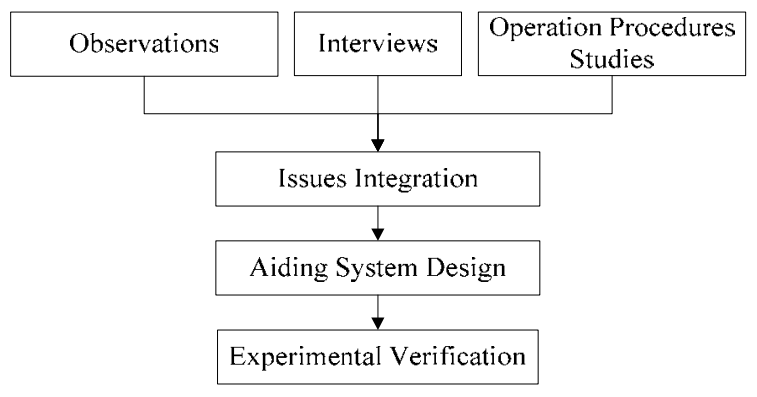

Figure 1 Flowchart for study process

\subsection{Issue of fault diagnosis in NPPs}

At the Lungman NPP, three kinds of procedures have been implemented to deal with an abnormal operation status, i.e., Emergency Operating Procedure (EOP), Abnormal Operating Procedure (AOP), and Annunciator Response Procedure (ARP). There are seven EOPs, about 80 AOPs, and over 20,000 ARPs. When the alarms occur, the first thing the operator must do is to confirm whether an EOP should be performed and, if so, which one. If the plant is at risk for radiation leakage, the operator will perform an EOP. If there is no appropriate EOP for the emergency situation at hand, the operator will consider an AOP next. If there is no proper AOP, the operator will operate alarms with ARPs. The entry conditions of EOPs are clearly defined and listed in the EOPs. In addition, each individual alarm has a special ARP. However, identifying AOPs is more difficult than identifying EOPs or ARPs. There are many abnormal symptoms listed in each AOP. All of the symptoms listed in the AOP might not necessarily be presented. Some symptoms may be related to each other, but some are independent of each other or mutually exclusive. The situation becomes more difficult when 
many AOPs have the same symptoms. The operators must pay consider many information sources, the organize information, and make a decision. The process of decision-making during identifying AOPs is very complex. How to help the operator to identify AOP accurately is an important issue.

\subsection{Development of an expert system}

The expert system was developed based on the issues described above. The main purpose was to reduce the amount of information the operator must pay attention and integrate during the diagnosis process and to prevent the operator from missing important information. In other words, the objective of fault diagnosis support system is to help operators identify AOPs easier and accurately than before.

An expert system could filter out unrelated information and AOPs and gather some symptoms related to the current event. In addition, it could provide the operator with some suitable AOPs. The operator could refer to the advice of the expert system and consider what to do next. According to the AOPs, there are many symptoms related to abnormal events, but it is not necessary to present every symptom listed in the AOPs. Moreover, since some symptoms might belong to several AOPs, the expert system can select the related AOPs when an unusual symptom occurs. After the similarity analysis, the operator can refer to the information for diagnosing the fault. Because all the diagnosis rules of the expert system are based on abnormal operating procedures, the more complete AOPs make the expert system more powerful.

\subsection{Constructing the abnormal symptom matrix}

It is straightforward to place the information sources of system level alarms, plant level alarms, plant status tiles, and individual alarms into "symptom occurs" and "symptom does not occur" categories. Using the attributes of the information, the abnormal symptom matrix can be constructed. In the abnormal symptom matrix, the "symptom occurs" category is designated by " 1 ", and the "symptom does not occur" category is designated by "0"(Table 1). After transformation, the data can be processed by the computer program.

\subsection{Experiment}

An experiment was conducted to verify the effect of the expert system. In NPPs, it is the best that the operator is able to make decisions quickly and correctly. However, making decision correctly is more important than making decision quickly. In order to make the subjects to perform task carefully as the operators who deal with alarm in an emergency, the subjects were asked to make a correct decision as the first priority, and to perform task quickly the second priority.

The experiment lasted about 50 minutes, and the data of decision time, error ratio, and NASATLX score were collected. The procedure of the whole experiment is as follows:

(1) Qualification test:

Thirty-two subjects were asked to classify the system level alarm to reactor-related, cooling system-related or power system-related. That information could help subjects diagnose and make decisions in the experiment. The formal experiment didn't start until the subjects were able to classify up to $90 \%$ system level alarm in the qualification test. (5 min)

(2) Experimental introduction :

The experimenter introduced experimental tasks. (5 min)

(3) Demo :

The aim of this step was to confirm that all subjects understand the diagnostic procedure completely. (5 min)

(4) Formal experiment :

The subjects were randomly assigned to perform tasks in one of two different modes (the original mode and the expert system mode) in the beginning. After finishing all the tasks, they were assigned to the other mode later. The data of decision time and the number of errors during the experiment were recorded. (10 $\mathrm{min})$

(5) Fill the NASA-TLX questionnaire :

The subjects filled out the NASA-TLX index after the experiment. (5 min)

(6) End of the experiment. 
Table 1

Partial of the abnormal symptom matrix of this study

\begin{tabular}{|c|c|c|c|c|c|c|c|c|c|c|c|c|c|c|c|c|c|c|c|c|}
\hline & RPS & RBSW & RBCW & SGT & LDI & ARM & PRM & CSTF & OG & RW & FPCU & ACS & DWC & SLC & $\mathrm{RCIC}$ & HPCF & RHR & RBHV & TBHV & ... \\
\hline 501.1 & 1 & 0 & 0 & 0 & 0 & 0 & 0 & 0 & 0 & 0 & 0 & 0 & 0 & 0 & 0 & 0 & 0 & 0 & 0 & $\ldots$ \\
\hline 501.2 & 1 & 0 & 0 & 0 & 0 & 0 & 0 & 0 & 0 & 0 & 0 & 0 & 0 & 0 & 0 & 0 & 0 & 0 & 0 & .. \\
\hline 501.3 & 0 & 0 & 0 & 0 & 0 & 0 & 0 & 0 & 0 & 0 & 0 & 0 & 0 & 0 & 0 & 0 & 0 & 0 & 0 & ... \\
\hline 501.4 & 0 & 0 & 0 & 0 & 0 & 0 & 0 & 0 & 0 & 0 & 0 & 0 & 0 & 0 & 0 & 0 & 0 & 0 & 0 & $\ldots$ \\
\hline 502.1 & 0 & 0 & 0 & 0 & 0 & 0 & 0 & 0 & 0 & 0 & 0 & 0 & 0 & 0 & 0 & 0 & 0 & 0 & 0 & ... \\
\hline 502.2 & 0 & 0 & 0 & 0 & 0 & 0 & 0 & 0 & 0 & 0 & 0 & 0 & 0 & 0 & 0 & 0 & 0 & 0 & 0 & ... \\
\hline 503.1 & 0 & 0 & 0 & 0 & 0 & 0 & 0 & 0 & 0 & 0 & 0 & 0 & 0 & 0 & 0 & 0 & 0 & 0 & 0 & $\ldots$ \\
\hline 503.2 & 0 & 0 & 0 & 1 & 0 & 0 & 0 & 0 & 0 & 0 & 0 & 0 & 0 & 0 & 0 & 0 & 0 & 1 & 0 & ... \\
\hline 504.1 & 0 & 0 & 0 & 0 & 0 & 0 & 0 & 0 & 0 & 0 & 0 & 0 & 0 & 0 & 1 & 1 & 1 & 0 & 0 & .. \\
\hline 505.1 & 0 & 0 & 0 & 0 & 0 & 0 & 0 & 0 & 0 & 0 & 0 & 0 & 0 & 0 & 0 & 0 & 0 & 0 & 0 & ... \\
\hline 506.1 & 0 & 1 & 1 & 0 & 0 & 0 & 0 & 0 & 0 & 0 & 0 & 0 & 0 & 0 & 0 & 0 & 1 & 0 & 0 & ... \\
\hline 507.1 & 0 & 0 & 0 & 0 & 0 & 0 & 0 & 0 & 0 & 0 & 0 & 0 & 0 & 0 & 0 & 0 & 0 & 0 & 0 & $\ldots$ \\
\hline 508.1 & 0 & 0 & 0 & 0 & 1 & 1 & 0 & 0 & 0 & 0 & 0 & 1 & 1 & 0 & 0 & 0 & 0 & 0 & 0 & .. \\
\hline 508.2 & 0 & 0 & 1 & 0 & 1 & 0 & 0 & 0 & 0 & 1 & 1 & 0 & 0 & 0 & 0 & 0 & 1 & 1 & 0 & $\ldots$ \\
\hline 508.3 & 0 & 0 & 0 & 0 & 1 & 0 & 0 & 1 & 1 & 1 & 0 & 0 & 0 & 0 & 0 & 0 & 0 & 0 & 1 & ... \\
\hline 508.4 & 0 & 0 & 0 & 0 & 1 & 1 & 1 & 0 & 0 & 0 & 0 & 0 & 0 & 0 & 0 & 0 & 0 & 1 & 0 & ... \\
\hline 508.5 & 0 & 0 & 0 & 0 & 1 & 1 & 1 & 0 & 0 & 1 & 0 & 0 & 0 & 0 & 1 & 0 & 0 & 1 & 0 & ... \\
\hline 508.6 & 0 & 0 & 0 & 0 & 0 & 1 & 0 & 0 & 0 & 0 & 0 & 0 & 0 & 0 & 0 & 0 & 0 & 0 & 0 & $\ldots$ \\
\hline 509.1 & 0 & 0 & 0 & 0 & 0 & 0 & 0 & 0 & 0 & 0 & 0 & 0 & 0 & 0 & 0 & 0 & 0 & 0 & 0 & $\ldots$ \\
\hline 509.2 & 0 & 0 & 0 & 0 & 0 & 0 & 0 & 0 & 0 & 0 & 0 & 0 & 0 & 0 & 0 & 0 & 0 & 0 & 0 & .. \\
\hline 509.3 & 0 & 0 & 0 & 0 & 0 & 0 & 0 & 0 & 0 & 0 & 0 & 0 & 0 & 0 & 1 & 0 & 0 & 0 & 0 & ... \\
\hline 509.4 & 0 & 0 & 0 & 0 & 0 & 0 & 0 & 0 & 0 & 0 & 0 & 0 & 0 & 0 & 0 & 0 & 0 & 0 & 0 & $\ldots$ \\
\hline 510.1 & 1 & 0 & 0 & 0 & 0 & 0 & 0 & 0 & 0 & 0 & 0 & 0 & 0 & 0 & 0 & 0 & 0 & 0 & 0 & $\ldots$ \\
\hline 511.2 & 0 & 0 & 0 & 1 & 1 & 1 & 1 & 0 & 0 & 0 & 0 & 0 & 0 & 0 & 0 & 0 & 0 & 1 & 0 & ... \\
\hline 512.1 & 0 & 0 & 0 & 0 & 0 & 0 & 0 & 0 & 0 & 0 & 0 & 0 & 0 & 0 & 0 & 0 & 0 & 0 & 0 & $\ldots$ \\
\hline 512.2 & 0 & 0 & 0 & 0 & 0 & 0 & 0 & 0 & 0 & 0 & 0 & 0 & 0 & 0 & 0 & 0 & 0 & 0 & 0 & .. \\
\hline 512.3 & 0 & 0 & 1 & 0 & 0 & 0 & 0 & 0 & 0 & 0 & 0 & 0 & 0 & 0 & 0 & 0 & 0 & 1 & 0 & .. \\
\hline$\vdots$ & $\vdots$ & $\vdots$ & $\vdots$ & $\vdots$ & $\vdots$ & $\vdots$ & $\vdots$ & $\vdots$ & $\vdots$ & $\vdots$ & $\vdots$ & $\vdots$ & $\vdots$ & $\vdots$ & $\vdots$ & $\vdots$ & $\vdots$ & $\vdots$ & $\vdots$ & $\because$ \\
\hline
\end{tabular}

\section{Results}

\subsection{The decision making time}

The decision-making time was the duration between when the alarm signal started and the subject completed her or his diagnosis and made a decision. The descriptive statistics were shown in Table .2. The results of the Wilcoxon signed-rank tests indicated that the median of the decision-making time of the original mode and the expert system mode under one abnormal event occurrence were significantly different $(Z=-2.206, p<0.05)$. In addition, the median of the decision-making time of the original mode and the expert system mode when two abnormal events occurred were significantly different $(Z=-3.740$, $\mathrm{p}<0.05)$.
Table 2

Descriptive statistics of decision-making time

\begin{tabular}{|c|c|c|c|}
\hline Modes & Conditions & Mean (sec) & S.D. \\
\hline \multirow{3}{*}{$\begin{array}{l}\text { Original } \\
\text { mode }\end{array}$} & One event & 74.44 & 43.05 \\
\hline & Two events & 75.29 & 24.92 \\
\hline & Total & 74.87 & 34.90 \\
\hline \multirow{3}{*}{$\begin{array}{l}\text { Expert Syst } \\
\text { mode }\end{array}$} & One event & 57.77 & 27.43 \\
\hline & Two events & 56.45 & 15.02 \\
\hline & Total & 57.11 & 21.95 \\
\hline
\end{tabular}




\subsection{The accuracy of decision making}

The result of the Wilcoxon signed-rank tests confirmed that the median of the error ratio of the original mode was not significantly different from the support system mode $(Z=-1.414, p=0.157)$. On the contrary, when two events occurred, the median of the error ratio of the original mode was significantly different from the support system mode $(Z=-2.588$, $\mathrm{p}<0.05)$. In the experiment, the accuracy of decision-making was about $51.6 \%$ with the original mode and $75 \%$ with the expert system. When both situations were combined, the median of the overall error ratio of the original mode was significantly different from the expert system mode $(Z=-2.911, \mathrm{p}<0.05)$. The accuracy was $65.5 \%$ with the original mode and $83.8 \%$ with the expert system.

\subsection{Subjective workload rating NASA-TLX}

A paired-sample $t$ test was conducted on the experimental data of subjective workload rating (NASA TLX scores). The average subjective rating of NASA TLX scores for operating in the original mode $(\mathrm{M}=$ $60.84)$ were significantly higher than operating in the expert system mode $(M=50.01)$. The result showed that the mean of differences between the two different operating modes was statistically significant $(\mathrm{t}=$ $3.02, \mathrm{p}<0.05)$.

\section{Discussion and Conclusion}

\subsection{Discussion}

The results show that the accuracy of decisionmaking with the expert system was higher than that with the original mode. Subjects with the expert system spent less time making a decision than those in the original mode, irrespective of whether one or two abnormal events occurred. Especially, when two abnormal events occurred, operating with the expert system reduced the decision-making time and also improved the accuracy of the diagnosis. However, there was no significant difference in the accuracy of the decision making between the two modes when only one abnormal event occurred. The reason might be due to the fact that one abnormal event is simpler to deal with, and the subjects were less likely to be confused. There were no significant difference in the number of mistakes between the two modes, but there were fewer miss errors in the expert system mode.

Concerning the mental workload, the subjective rating on NASA-TLX in the expert system mode was significantly lower than that in the original mode. It is difficult for an operator to make a correct decision when he or she is working in a situation that imposes a high mental workload. That is why almost all the subjects' performances with the expert system were better than those with the original mode.

\subsection{Conclusion}

The results of experiment verified that the subjects' decision times were reduced significantly when they were assisted by the expert system. Almost all participants took less time in identifying the AOPs when an abnormal event occurred, which left additional time for the operator to deal with abnormal event. In addition, the errors of procedure selection and subjects' mental workload can be reduced with the assistance of the expert system. The most important observation is that the expert system can help people avoid overlooking important information when several abnormal events occur simultaneously. In conclusion, we highly recommend the expert system because it is helpful in identifying the AOPs and enhancing operation safety.

\section{Acknowledgment}

This study was supported by the ROC Atomic Energy Council and the ROC National Science Council, project no. NSC99NUE007008. The authors would like to thank the administration of Lungman Power Plant in Taiwan providing valuable information.

\section{References}

[1] J. M. Noyes and M. Bransby, People in Control: Human Factors in Control Room Design, 2001, pp.207-221.

[2] R. Ma, D.B. Kaber, J.M. Jones, R.L. Starkey, Team situation awareness in nuclear power plant process control: a literature review, task analysis and future research. In: In: ANS Fifth International Topical Meeting on Nuclear Plant Instrumentation, Controls, and Human Machine Interface Technology, Alberquerque, 2006, November 12-16.

[3] D.N. Hogg, K. Folleso, F.S. Volden, B. Torralba, Development of a situation awareness measure to evaluate advanced alarm systems in nuclear power plant control rooms. Ergonomics 38 (11), 1995, pp.2394-2413.

[4] K.J. Vicente, N. Moray, J.D. Lee, J. Rasmussen, B.G. Jones, R. Brock, T. Djemil, Evaluation of a Rankine cycle display for 
nuclear power plant monitoring and diagnosis. Human Factors 38 (3), 1996, pp.506-521.

[5] A. Isaac, S. T. Shorrock, B. Kirwan, Human error in European air traffic management: the HERA project. Reliability Engineering and System Safety, 75, 2002, pp.252-272.

[6] K.C. Kwon, J.H. Kim, Accident identification in nuclear power plants using hidden Markov models. Engineering Applications of Artificial Intelligence 12, 1999, pp. 491-501.

[7] S.J. Lee, K. Mo, P.H. Seong, Development of an Integrated Decision Support System to Aid the Cognitive Activities of Operators in Main Control Rooms of Nuclear Power Plants. In: Proc. of IEEE Symposium on Computational Intelligence in Multicriteria Decision Making (MCDM), 2007,pp. 146-152.
[8] H. Yoshikawa, Human-machine interaction in nuclear power plants, Nuclear Engineering and Technology, Vol. 79, no. 2, 2005,pp. 151-158

[9] J.T. Kim, K.C. Kwon, I.K. Hwang, D.Y. Lee, W.M. Park, J.S Kim, S.J. Lee, Development of advanced I \& $\mathrm{C}$ in nuclear power plants: ADIOS and ASICS. Nuclear Engineering and Design 207, 2001,pp. 105-119. 